\title{
UEBER DAS VERCELLIBUCH.
}

Von den vier, für kenntniss der angelsächsischen dichtung wichtigsten handschriften, des Beowulf, des sog. Cædmon, des Exeterbuches und der Vercellihs., wurde die letztgenannte am spätesten bekannt.

In Vercelli selbst wusste man, dass eine ziemlich umfangreiche handschrift vorhanden sei, welche, obgleich die buchstaben lateinische, doch in einer unverständlichen sprache geschrieben sei. Man rermutete allerdings, dass man eine angelsächsische handschrift hätte. Nachdem man aber dieselbe einigen gelehrten Engländern vorgelegt hatte und dieselben sie nicht lesen konnten, scheint sie ziemlich in vergessenheit geraten zu sein.

Das verdienst nun, zuerst auf dieselbe nicht nur aufmerksam gemacht, sondern auch zugleich eine abschrift eines teiles derselben genommen zu haben, gebührt einem deutschen juristen, Prof. Dr. Fr. Blume. Dieser gelehrte hielt sich vom 20. März 1821 bis 11 . Oktober 1823 in Italien auf und besuchte dort eine menge bibliotheken. 1822, 27. Oktober bis 19. November (nach eigener angabe), hielt er sich zu Vercelli auf. Das reiche ergebniss dieser reise wurde 1824 veröffentlicht im

I ter Italicum. Von Dr. Fr. Blume. Berlin u. Stettin. 1824-36. Ueber unsere hs. sagt er hier s. 99:

'Das andere buch (cod. CXVII) enthält legenden oder homilien in angelsächsischer sprache. Dies ist um so merkwürdiger, da keine kapitularbibliothek in Italien andere als lateinische oder italienische handschriften enthält; selbst griechische finden sich nur in Verona, und vielleicht in Ravenna'.

Ferner wurde nachricht darüber gegeben von Pertz, nach Blume's bericht, im

Archiv für ältere deutsche Geschichte. Bd. V (enth. Pertz:

Italiänische reise) $535 \mathrm{ff}$. Hannover 1824 . 
Zehn jahre nach der entdeckung erschien wider eine nachricht uber unsere hs. von Blume im

Rheinischen Museum für Jurisprudenz, jahrg. 1532. Göttingen 1833. IV, s. $234 \mathrm{ff}$.

Endlich erschien Göttingen 1834:

Bibliotheca Librorum Manuscriptorum Italica. Indices Bibliothecarum Italiæ... in Supplementum Itineris Italici congessit Fridericus Blume.

Hier findet sich s. 6 die nachricht aus Vercelli:

Bibliotheca Capituli. Homiliarum liber lingua anglosaxonica elegantissime scriptus. Fol. Cod. CXVII.

Blume gibt als probe:

De purificatione sancloe Marice. Men sæged us and myngath this halige godspel bevyrre arvyrdhan tide, the ve nu to dæge gode ælmihtigum to lofe and to are vyrdhiath, thæt irre (is). ${ }^{1}$

Es folgt darauf die übersetzung dieser zeilen in's Deutsche. -Weiterhin gab auf eigne betrachtung der hs. nachricht über dieselbe geheimrat Neigebauer: Die Bibliothek des Erzbischöflichen Domcapitels zu Vercelli in no. 12 vom

Serapeum. Zeitschrift für Bibliothekwissenschaft, Handschriftenkunde und ältere Literatur. Herausg. von Dr. Rob. Naumann. Achtzehnter Jahrgang. Leipzig 185\%.

Dieser aufsatz (s. 189 ff.) enthält über die hs. nichts neues. Nur sei hier der dort erwähnten ansicht des gelehrten Italieners Gazzera gedacht: Johannes Scotus Erigena habe sich in Vercelli aufgehalten und durch ihn sei die hs. nach Vercelli gekommen. ${ }^{2}$

Nachdem Blume angegeben hatte, dass sich auch angelsächsische gedichte $\mathrm{zwischen}$ den homilien der hs. fänden, wollte man in England möglichst bald den neuen fund den freunden der angelsächsischen literatur zugānglich machen. Kemble berichtet dartuber in seiner unten angeführten ausgabe s. V:

In the hope of bringing these valuable remains to England, and putblishing them here, I set out in the summer of 1834 for Vercelli; but having spent some months in traversing (xermany, I found myself, at the commencement of winter, still on this side the Alps, and cut off from all hope of crossing them by the storms which had broken up the passes. On returning to England, however, I found that one portion

1 Man vgl. unten fol. $90^{\mathrm{b}}(17)$.

2 Dieser aufsatz enthält ausser unendlich vielen druckfehlern auch einige sachliche irrtïmer. Ueber Kemble's ausgabe vgl. unten. Falsch ist, dass die gedichte wie Andreas und Elene 'auf solche angelische homilien' (wie sie die hs. enthält) gegründet seien, falsch, dass Kemble nur Andreas, nicht auch Ëlene veröffentlicht hätte (vgl. unten). 
of my plan was already executed. 'The then existing Record Commission had employed Dr. Blum ${ }^{1}$ to copy the Manuscript, and had caused the poems to be extracted and printed under the care of Mr. Thorpe.

Die erste auswabe der in unserer hs. enthaltnen gedichte geschah in:

Appendix B. to Mr. Cooper's Report for 1836 .

Ueber die entstehung des werkes finden sich in demselben gar keine angaben. Nach Kemble's aussage aber fertigte Blume im auftrage der Record-Commission am anfange der dreissiger jahre eine abschrift der gedichte an und Thorpe leitete den druck für die Record-Commission. Es wurde das ganze als Appendix $B$ zu Cooper's Report über Rymeri Foedera gedruckt. Dies war also die erste ausgabe der gedichte der Vercelli-hs. Ueber die homilien wurde bisher iiberhaupt nichts eingehenderes veröffentlicht.

Cooper's werk wurde nur in 250 exemplaren ausgegeben. Eincs davon, welches Lappenberg besass, benutzte Jak. Grimm zur ausgabe der zwei umfangreichsten gedichte der hs., von Andreas und von Elene:

Andreas und Elene. Herausg. von Jakob Grimm. Cassel 1840.

Schon bald nach dem erscheinen des Cooper'schen werkes empfaud man auch in England das bedürfniss nach einer neuen, leichter zugänglichen ausgabe. So entstand:

The Poetry of the Codex Vercellensis, with an English Translation. By J. M. Kemble. Part. I The Legend of St. Andrew. Part. II Elene and Minor Poems. London, Printed for the Elfric Society 1544 und 1846 (no. 5 und no. 6 der veröffentlichungen der Exlfric Society).

Aus der oben angefiihrten stelle aus Kemble's vorrede ergibt sich also, dass Blume eine abschrift anfertigte, welche, ohne neue vergleichung, von Thorpe und Kemble benutzt wurde.

Eine neue vergleichung wurde seitdem nur für Elene noch angestellt von Prof. P. Knöll in Wien und veröffentlicht in:

Cynewulf's Elene. Mit einem Glossar heransgegeben von Julius Zupitza. Berlin 1877.

So wurde es für mich ganz unerlässlich notwendig, für meine neuausgabe der Bibliothek der ongelsächsischen Poesie eine vergleichung der hs. an ort und stelle vorzunehmen. Es geschah dies im herbste 1881 .

1 So wird der name durchgängig von Kemble geschrieben. 
Das ergebniss der vergleichung ist: die in der hs. enthaltenen dichtungen sind alle bereits veröffentlicht. Es stehen also keine andren etwa zwischen den prosahomilien verborgen. Eine menge kleiner ungenauigkeiten kann ich berichtigen, im ganzen aber haben Blume und Knöll sehr gut gelesen und getreu abgeschrieben.

Genauere angaben über die hs. und über versehen der herausgeber der gedichte werde ich im 2. bande der Bibliothek bringen. ${ }^{1} \mathrm{Da}$ aber die herausgabe der noch ganz unbekannten prosahomilien in einem bande der Bibliothek der angelsächsischen Prosa wol noch längere zeit auf sich warten lassen wird, will ich hier genaueres uber die homilien der Vercellihandschrift geben.

Ich benutze diese gelegenheit gerne, um dem archivar der capitelbibliothek zu Vercelli, herrn canonicus Canetti, hicr meinen besten dank für die grosse freundlichkeit, mit der er mir entgegenkam und meine arbeiten förderte, auch öffentlich auszusprechen.

\section{Die homilien des Vercellibuches.}

Auf dem ersten blatte der handschrift sind noch einzelne buchstaben zu lesen, allein trotz der in früherer zeit (von Blume?) angewanten reagenzien sind keine grösseren stücke mehr zu entziffern. Auch das jetzt mit fol. 1 bezeichnete blatt war sicherlich kein ursprünglicher anfang der hs.

Auf bl. 2 ff. steht eine predigt über die leiden Christi, seine grablegung und höllenfahrt (1). Sie beginnt mitten im satze und worte:

scua (?) pe . . . pan ne meaht du pa prowunze zelettan

pe fæder wolde .7 zeteohod hæfde pæt ic for man

cynnes hælo zeprowian sceolde. Sazap oðer zod

spellere pæt he ure dryhten æt hrine pæs mannes

earan 7 he wæs sona æfter pain hal zeworden. Đa hie

pa crist oferfanzenne hæfdon. pa zebundon hie

hine . 7 læddon ærest to annan. wæs se anna sweor

pæs caifan pe $\delta y$ zere wæs bisceop etc.

1 Soviel sei nur auch hier schon beunerkt: Herr canonicus Canetti wusste nichts genaues darüber anzugeben, wie die hs. nach Vercelli kam. Doch da ziemlich frühe ein hospiz für angelsächsische pilger in dieser stadt errichtet worden ist, so mag aus diesem das buch in die bibliothek übergegangen sein. 
Schluss auf $9^{\mathrm{a}}$ :

7 us pa

zife forzeaf pcet nu nis nænezum $\overline{\mathbf{m}}$ pearf pat he helle sece ac he bio sona to ecre reste zelæded syppan he pis deap lice lif forlæte $\partial$ if he nu her on worulde sog 7 riht in his life don wile. Ac utan we nu forpan a sinzalice ussum dryhtne hælendan criste panc seczan eallre pære earmodnesse pe se heofonlica cyninz æelmihtiz drihten In pas halzan tîd eallum man cynne zecyðde. Utan we hine nu lufizan ofer eall odre pinz swa we nu zehyrap paet he us lufode 7 us hyldo to forhte pas him sie lof 7 wuldor a to widan feore $a$ in ecnesse purh eallra worulda woruld AMEN.

a butan ende.

$9^{\mathrm{b}}$ oben beginnt eine predigt ïber das jüngste gericht (2):

\section{MEN ĐA LEOFESTAN}

pæs myclan domdæzes worc bið swiðe ezeslic 7 andrys lic eallum zesceaftum. In pam dæze pa hleoðriendan lizeas forbær nap pane blodzemenzdan zeard 7 pa pe nu her syndon on myclum zylpe 7 on unny,tre zesyhye zoldes 7 seolfres 7 zod webbes 7 wozzestreona ac we sint nu pam zeliccost for truwode pe he us noto (!) ne cyme 7 on pam dæze gewit sun nan leoht 7 monan leoht 7 pa leoht ealra tungla: 7 on pam dæze bið dryhtnes rod blode flowende betweox wolcnum

Schluss auf $12^{\mathrm{a}}$ :

Utan we nu efstan to pan pa hwile pe we ura weza wealdan moton pær hie næfre leofe zedalap ne lape zesamniap ne næfre dæz ne cymeð æfter dæz̄ ne niht æfter nihte . ac pær bið ece leoht 7 blis 7 ece wuldor 7 ece zefea mid urum dryhtne middanzeardes alysende pæt is efne pe ilca zod se de leofap 7 ricsap mid fæeder 7 mid suna 7 mid pam hal ${ }^{2}$ an zaste pam is wuldor 7 wyromynd purh ealra worulda woruld aa butan ende. aĩu.

$12^{\mathrm{b}}$ beginnt eine predigt, welche giter der wahro christ besitzen muss (3):

BRoðer pa leofestan ic cyðe pæt preo pinz synt ærest onforeweardum $æ$ hhwylcum men nyd behefe to habbanne .I. is z̆leafa . Oðer is hiht . pridde soð lufu . On Jam zeleafan is poet he zelyfe on zod fæder almihtizne. 7 on sunu .7 pone halzan zâst . 7 on pa todældan prynesse 7 on pa purh wuniendan annysse $u$. s. $w$.

Schluss bl. $16^{\mathrm{a}}$ :

$$
\text { witod }
$$

lice preo cyn synt ælmessena an is lichamlic poet is poet man pam wædliendan sylle to zode poet he mæze oðer is zastlic poet is poet man forzife pam pe wið hine żylteð 
pridde poet man pam zyltendan żstyre $7 \partial_{1}^{\mathrm{a}}$ dwoliendan an rihtan zebrinze. pas pinz us $\bar{z}$ dafenar $\bar{z}$ fellan mid fæder 7 mid suna 7 mid pam halza(n) zaste $\hat{\mathbf{a}}$ in ecnesse purh ealra worulda woruld aa bu tan ende. AMEN.

$16^{\mathrm{b}}$ beginnt eine predigt über jüngstes gericht und ähnliche gegenstände (4):

MEN pa leofestan Ic eow bidde 7 earınodlice lære; pat ze wepen 7 forhtien on pysse medmiclan tide for eowrum synnum forpan ne bioð cowre tearas 7 eowre hreowsunza for noht zetealde on pære toweardan worulde $u$. s. $w$.

Schluss auf $24^{\mathrm{b}}$ :

Is us poñ mycel pearf . men . pât

pæt we zezanzen pæs hælendes scylde near 7 pæs diofles stræle fyr 7 lufien we urne dryhten zod swa us oft be boden is poñ zif $\gamma$ he us ece lif In his wuldres fæzernesse pær he leofar 7 ricsar In ealra worulda woruld a butan ende ameN;

Auf $25^{\text {a }}$ beginnt eine predigt über die geburt Christi, mit besonderer überschrift versehen (5):

To middan wintra. Ostende not dñe.

HER sezð pis halize zodspel be pære hean medomnesse pisse halzan tide pe nu onweard is 7 us læreð pætte we pas halzan tiid zedefelice 7 clænlice weoryien zodes naman to lofe and to wuldre.

Schluss auf $29^{\mathrm{a}}$ :

7 we bioð butan synna wommum 7 bliðe butan un rotnesse 7 we lifzende butan deaðe zastlice herizen we ures dryhtnes naman hælendes cristes he leofad 7 ricsað ââ butan ende .7

Auf $29^{\mathrm{b}}$ beginnt das gedicht von Andreas. Es geht bis auf $52^{b}$.

Dann folgt auf $52^{\mathrm{b}}$ das gedicht von den schicksalen der zwölf apostel. Thorpe und Kemble halten es für unvollständig am schlusse. Es schliesst gerade unten auf bl.53'. Es können also einige zeilen, welche auf dem neuen blatte standen, verloren gegangen sein. Viele aber waren es keinesfalls, dies beweist der inhalt der letzten verse, die eine bitte des dichters, für ihn zu beten, enthalten.

Auf bl. 54a folgt eine predigt iiber die wunder vor Christi geburt und auf der flucht nach Aegypten. Auch hier steht eine besondere uberschrift (6): 
INcipit narrare miracula que facta fuerant ante adu(en)tum

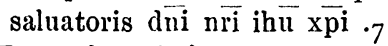
HER sazad ymb das mæran zervyrd pe to pyssum dæze ze weard pætte ælmihtiz dryhten sylfi pus world zesohte 7 purh unwemme fæmnan on pas world acenned wæs to pan pæt he eall manna cyn fram hellwara wite alysde 7 to heofona rices wuldre zefremede.

Schluss auf $56^{\mathrm{a}}$ :

Hwæt we nu zehyrdon seczan hwylene hwezu dæl ymb usses dryhtnes zebyrd swylce eac ymb pawun (!) pe he on his cild hade utan we nu eorne tilian pæt we pe selran syn poñ we py] leca bysena usses dryhtnes beforan us reccan 7 rædan ze zehyrad utan we healdan sybbe 7 lufan betwiohs us ponne zyldeð us zod ece mede æt ussum ende.

Auf $56^{\text {b }}$ beginnt eine neue predigt ohne iiberschrift (7): BV'IAN tweon lar is haliz domes dæl 7 ealles swiojost zif hio hyre zyme leste fram adrifed 7 ælce zitsunze afyrreð 7 pyssa woruldlicra pinza lufan zewaniz 7 poet mod to

Die predigt reicht bis auf $59^{a}$ (dieses blatt wurde fälsehlich als $49^{\mathrm{a}}$ bezeichnet). Die letzten zeilen von $58^{\mathrm{b}}$ bis $59^{\mathrm{a}}$ oben lauten:

Ac mid zemetezunze zefiæte

wizen 7 us fram awiorp pa wol 7 jearnien 7 onfon pa zod pe us zehatene synt on pam hælendan criste 7 mid pam halezan zaste In ealra worulda $49^{\text {a }}$ (st. 59a) woruld .7

Dann beginnt eine neue predigt ohne überschrift, wie auch die zwei folgenden (8):

MEN $\boxplus A$ leofestan manad us 7 myndzad on pyssum bocum Scs zrezorius se haleza writere se $\partial$ is zewrit sette 7 wrat pat we ymb us sylfe eorne pencen liered he us pat we sien zemyndize Schluss auf $61^{\mathrm{a}}$ :

mid

soðum zodes prowerum 7 mid eallum sorfæstum 7 zecorenum Jam zodes suna $\gamma_{a m}$ pe aliofað 7 rixap mid fæder 7 mid suna a to widan feore AMEN.

Neue predigt beginnt $61^{\mathrm{a}}(9)$ :

MEN ta leofestan manad us 7 mynzap peos halize boc pæt we sien zemyndize ymb ure sawle pearfe 7 eac swa ures pæs nehstan dæzes 7 pære tosceadenesse ure sawle ponne his of Jam Schluss auf $65^{\mathrm{a}}$ :

$$
\text { zif we pænne swa don wyllad }
$$

[Rasur] swa us dryhten beden bafad ponne moton we mid him 7 mid his pam halezan zæste wunizean In ealra worulda woruld. AmeN.

Auf derselben seite beginnt eine neue predigt (10): HER sazad on pyssum halezum bocum be ælmihtizes 
dryhtnes zodspelle pe he him sylfum purh his $\gamma \mathrm{a}$ halezan mihte zeworhte mannum to bysene 7 to lare Schluss auf $71^{\mathrm{a}}$ :

enzlas on heofenum 7 we syndon pyder zeladode 7 ze hatene to pan halezan 7 to pam cynelycan friostole pær drihten crist wunap 7 rixað mid eallum halezum a butan ende. ameN.

Es folgen nun drei predigten für die drei 'zanzdazas', d. h. für die drei bet- und fasttage, welche dem himmelfahrtsfeste vorausgehen. Die erste beginnt $71^{\mathrm{b}}(11)$ :

Spel to forman zanzdæze ${ }^{1}$

MEN ${ }^{\prime}$ 'a leofestan pis syndon halize dazas 7 halivendlice 7 ussum sawlum læcedomlice 7 us zerised pet we hie wel be zanzen mid fæstenum 7 mid zebedum 7 mid reliquia socnum Schluss auf $73^{\mathrm{b}}$ :

pysse halzan tide tilien we nu foryan pæt we hie zedeflice to ðam bezanzen mid zastlicum mæzenum pe jær to zeset is se zod us zefultumize pe ofer us ealle liofad 7 rixar AmeN.

$73^{\mathrm{b}}$ beginnt $(12)$ :

Spel to Oam oðrum zanzdæze ${ }^{1}$

JIR'sandæz (!) we wæron manode men pa leofestan pissa haliza (!) daza bizanznes liornodon we pæt zeo hædene liode hæfdon pry dazas synderlice beforan hira odrum zewunan pæt hie onzuldon Schluss auf $75^{\mathrm{b}}$ :

pæt we Jonne ealle pe selran sien ze onwearde ze ofwearde pæs zodspelle .7 pa soðan lufan zodes 7 manna eorne healden arwior lice eallum tidum usses lifes zif we jus don willad ponne ze earn ize we us zodes miltse 7 blisse 7 æfter pysse worulde êce are 7 reste mid him 7 mid his halzum a in ealra worulda woruld on ecnesse. 7

$75^{\mathrm{b}}$ beginnt $(13)$ :

Spel to priddan zanzdæze ${ }^{1}$

ĐIS IS $^{2}$ se pridda dæz men pa leofestan pysse halzan tide pe us on swiðe wel zelimped pæt we ealle eadmodlice sculon dryhtne peowian

Schluss auf $76^{\mathrm{b}}$ :

pæt we his pa halzan lare zehyren swa we nu dydon pæt we syn pe beteran 7 pe selran for bæm lifum in ealra worulda world to widan feore mid fæder 7 mid suna 7 mid pam halzan zaste In ecnesse Amen. larspel to swylcere tide swa man wile ${ }^{3}$ (14) MEN ta leofestan pis synt halize dazas 7 zastlice 7 ussum sawlum læcedomlice 7 we micle nydpearfe habbad pat we $\partial æ t$ zeornlice zepencen 7 zemynen pa hwile pe we pære tide

1 Mit roter schrift.

2 Mit roter schrift. Das erste IS steht im $\boxplus$ drin.

3 Mit roter schrift. In der selben zeile mit Amen. 
Bl. 77 hat sehr stark durch anwendung von reagenz gelitten. Die predigt schliesst $80^{\mathrm{b}}$ :

witu 7 on pa ecan forwyrd zescyrede Ac paet we moton zefeonde faran mid urum dryhtne 7 mid his enzlum 7 mid callum zodes halzum on pone heofenlican ejel 7 pæs siojoun brucan on ecnesse amen 7

Auf $80^{\mathrm{b}}$ beginnt eine neue predigt (15):

ALIA OMELIA INE DIE IVDICII.'

MI sæż us on pyssum bocum hu se halza thomas zodes apst acsode urne dryhten hwænne antecristes cyme wæere $\partial a$ wæs drybten sprecende to him 7 бus ewæ pat hit sie on Jam nexstan tide.

Bl. 84 ist sehr stark durch reagenz beschädigt. Auf $85^{\text {b }}$ schliesst obige predigt.

7 pær syððan wuniað In ecum wul

dre 7 hie habbað symle zefean sydðan 7 blisse mid urum dryhtne $\partial \mathrm{am}$ sie symble wuldor 7 wyromynd 7 ece zefean a butan ende In sc̄a sciorum ameN ${ }_{7}$

Auf demselben blatte beginnt (16):

OMELIA EPYHFANIA DN $\bar{N} .^{1}$

MI sceolon we nû hwylcum hwezu wordum seczan be خære arweoronesse pysse halzan tide 7 pysses halzan dæzes . Jæt wæs on pyssum dæze be nu 7weard is $\partial æ t$ ure dryhten zefulwad wolde bion.

Bl. $86^{a}$ ist sehr stark durch reagenz beschädigt. Schluss auf bl. $90^{\mathrm{b}}$ :

7 mid pyllicum dædum

we mazon pa heofonlican rice bezitan 7 on becyman

7 mid urne drybten hælende crist se de leofad 7

rixað nû $\hat{a}$ purh eallra woruld $\hat{a}$ woruld $\hat{a}$ butan

ende In sclä sclōrum ameN.

Noch auf $90^{\text {b }}$ beginnt (17):

DE PVRIFICATIONE SCA MARIA (!)!

III sæzeð us 7 mynzap pis halize zodspel be pysse arwyrðan tide pe we nu to dæze zode ælmihtizum to lofe 7 to are

91 a wyrðiap pæt is se feowertiza dæz nu todæz ures dryhtnes hælendes cristes acennesse u. $s . w$.

Mit $91^{\text {a }}$ beginnt eine andere hand.

Der schluss dieser predigt steht auf $94^{1}$ :

$$
7 \text { we pær habbað â soðe sybbe } 7 \text { soðe }
$$

lufan In ecnesse 7 soðne zefean 7 ecne eard 7 ece wuldor mid hine 7 mid his pam halzum $\hat{a}$ in eallra woruld a woruld a butan ende in sīa sclōrum a⿳亠丷⿵冂. 7

1 In schwarzer schrift. 
Noch unten auf $94^{\mathrm{b}}$ steht (18):

DE SCO MARTINO CONF.'

95 a $\bar{M}$ Mazon we nu hwylcum hwezo wordum aseczan

be pære arwyronesse pysse halzan tide

7 be pære arwyrdan zebyrde 7 be pæes halzan

bisceopes pysses eadizan weres pe we nu In and

weardnesse his tid weordiad 7 mærsiad pe marti

nus wæs haten wæs he zode swiðe zecoren on his

peawum was he in pannana pære mæzðe In woruld cumen In arrea $\gamma_{a m}$ tune.

Die handschrift verschlechtert sich nun immer mehr, auf $101^{\mathrm{a}}$ ist sie abscheulich, $101^{\mathrm{b}}$ wird sie wider besser.

Das leben Martin's schliesst $101^{\mathrm{a}}$ :

$\bar{M}$ pæt we pæs halzan weres scs mar

tinus lif 7 his dæda onherien pæs pe ure ze

met sie 7 wuton hine biddan pæt he us sie

in heofonum pinzere wio urne dryhten nu we

her on eoryan hine zeond middanzeard wyr

Jiap dryhten us to pam zefultumize se 犭e

leofap 7 rixap ââ In eallra worulda woruld a bu-

tan ende ameN.

Auf $.101^{\text {b }}$ beginnt das gedicht: Rede der seele an den leichnam. Es ist uns nur als bruchstück erhalten. Unten auf $103^{b}$ bört es auf. $104^{\text {a }}$ beginnt neues blatt und ein neues gedicht, dessen anfang aber fehlt. Der schluss desselben steht auf $104^{\mathrm{b}}$. Grein nennt es Be monna lecise. Auf derselben seite steht der anfang von der Vision vom kreuze. Dieses gedicht schliesst unten (mit der 32. zeile von) $106^{\mathrm{a}}$. Es ist offenbar vollständig erhalten. $106^{\mathrm{b}}$ wird mit den homilien fortgefahren. Die folgenden homilien haben keine besondere ubberschriften.

$106^{\mathrm{b}}$ beginnt (19):

MEN $\partial a$ leofestan us zedafenap ærest

pæt we zemunen 7 zereccen be zode

ælmihtizum pe zeworhte heofonas 7 eorðan

7 ealle zesceafia pone we sculon zelyfan prynlic,

7 anlicne on spede; oð̀er is soðlice se hâd ælmihtizes fæder

Oðer is ælmihtizes suna oðer ys ælmihtizes halizes zastes

Die predigt schliesst auf $109^{\mathrm{b}}$ :

pær is ece med 7 pær is lif butan deaðe 7 zeozor butan ylde

7 leoht butan pystrum 7 zefea butan unrotnesse 7 sybb bu tan unzepwærnesse 7 orsorhnes butan deapes eze to

1 In schwarzer schrift. 
lybbenne $7 \mathrm{pær}$ is ece zesæliznesse mid fæder 7 mid pam suna 7 mid pam halizan zaste. $\hat{A}$ butan ende ameN : 7

Auf derselben seite $109^{\mathrm{b}}$ beginnt eine neue predigt (20):

MENN $\boxplus A^{1}$ [Vor Menn ist erst $M$, dann ME radiert] leofestan pis syndon halize dazas 7 hal wendlice 7 urum sawlum læcedomlice 7 us zedafenap pæt we hie wel bezanzen mid fæstenum 7 mid ælmes dædum 7 mid halizum zebedum 7 mid reliquia socnum $7 \mathrm{mi}^{\mathrm{d}}$ urum eadmodlicum zanze 7 mid eallre beortan onbryrdnesse 7 uton lætan ure idelan spræca.

Der schluss lautet $112^{\mathrm{a}}$ :

7 pær ys zefea

butan unrotnesse $7 \mathrm{pær}$ is leoht butan pystrum 7 Jær is wlite butan awendendnesse $7 \mathrm{pær}$ is ece blis 7 ece zefea mid pam ecan fæder 7 Jam efenêcan suna 7 mid his pam efenecan halizan zaste â butan ende $\cdot 7$

Auf $112^{\text {a }}$ beginnt eine neue homilic (21): MEN ĐA LEO

festan us ys mycel pearf pæet

we zod lufien of eallre heortan 7 of eallre sawle 7 of eallum mæzene 7 sydðan ure pa nehstan pret syndon calle cristene menn utan hie lufian eall swa us sylfe.

Schluss auf $116^{\mathrm{b}}$ :

pam pe he is ece dryhten 7 zefyllednes 7 fulfremednes eallra halizra se leofad 7 rixap mid fæder 7 mid suna 7 mid Jam halizan zaste on wuldre 7 on wyrömynde ââ butan ende on ecnesse . 7

Es folgt nun auf $116^{\mathrm{b}}$ die letzte homilie ohne überschrift, doch ist eine zeile leer gelassen (22):

HER sæzठ hu scs isodorus spræc be خære sawle zedale 7 be pæs lichoman. He cwæò min sawl on nearunesse is zeseted 7 min zast me hatad 7 min heorte is zedrefedu 7 mines modes nearunesse me nâtt eallum yflum ic eom seald . cwæò seo synfulle sawl 7 eallre unzesæliznesse ic eom bewrizen.

Schluss auf $120^{\mathrm{b}}$ :

se lichoma læmen is fordam pe he of Jam zeworht wæs 7 he eft to duste zeweorðan sceal. Ac uton we $\overline{\mathrm{m}} \partial_{\mathrm{a}}$ leofestan cW se halza ysodorus (!) eadmodlice biddan zod pæt he us zehealde her on worulde 7 on pære toweardan se de leofad 7 ricsad ầ butan ende In ecnesse.

Auf $121^{a}$ beginnt Elene und reicht noch ein stiuck auf $133^{b}$. Dann steht ohne iiberschrift ein stick aus einem prosaleben

1 A steht im $Đ$. 
des Gû̉lâc. Dass der jetzige anfang nicht der ursprüngliche war, beweist 'sprecenan' in der ersten zeile. Es ist dieselbe darstellung dieses heiligenlebens, welche sich vollständig Vesp. D XXI findet und von Goodwin herausgegeben wurde. Ich gebe einige proben mit dem Cotton texte, nach Goodwin, zum vergleiche.

Die Vercellihandschrift beginnt $133^{\mathrm{b}}$ :

Wæs pær in pam sprecenan izlande sum mycel hlæw of eorpan ze worht pone ylcan hlæw iu zeara men bræcon 7 dulfon for feos pinzım da was pær on oð̀re sidan ðæs hlæwes zedolfen swylce mycel seap on pam seape ufan se eadiza wer zuòlac him hus 7 eardunzstowe zetimbrode sona on fruman pæs de he pot ancer setl zesæt pa pohte he pæt he nawðer para ne wyllenes hæzles ne linenes brucan wolde ac on fellenum zezyrlan pat he wolde ealle dazas his lifes alifizean.

Goodwin s. 26:

Wæs pær on pam ealande sum hlaw mycel ofer eorðan ze worht, pone ylcan men iu zeara for feos wilnunza zedulfon and bræcon . pa wæs pær on opre sidan pæs hlawas zedolfen swylce mycel wæter-sead wære . On pam seade ufan se eadiza wer Guthlac him has zetimbrode, sona fram fruman pæs pe he pæt ancersetle zesæt . pa zepohte he pæt he nador ne wyllenes hræzles ne linenes brucan nolde, ac on fellenum zezyrelan pæt he wolde ealle his dazas his tifes alifian.

Zur vergleichung stehe hier noch das stück, wie die teufel Gûðlâc entführten.

$135^{\mathrm{b}}$ :

pa on pære nihte stilnesse zelamp semninza pat pær com micel mænezo para werizra zasta 7 hie eal poet hus mid heora cyrme zefyldon 7 him on ælce healfe Inzuton ufan 7 neopan 7 æzhwænon wæron hie onsyne ezeslice 7 hæfdon heafdu 7 lanze sweoran 7 mænizre onsyne wæron fulice.

$135 \mathrm{a}$ :

7 orfyrme on heora bearde 7 hæfdon ruze earan 7 woh neb ezeslice eazan 7 ondrysenlice muð̃as 7 heora teð wæron horses tuxum zelice $7 \mathrm{him}$ wæron pa hracan lize afylled 7 hie wæron on drysenlice on stefne 7 hie hæfdon wô sceancan 7 micle cneowu 7 hindan zreate 7 misscrence tan 7 has hrymedon on heora. cleopunze 7 hie swa unzemetlice hrymdon 7 foran mid forhtlicum ezesum 7 unzepwærnessum peet hit puhte paet hit eall be tweoh heofone 7 eorठ̀an hleoðrode pam ezeslicum stefnum næs pa næniz yldinz to pam syðdan hie In poet hus comon hie ta sona pone halzan wer eallum limum zebundenum hine tuzon 7 læddon ut of Jære cytan ond hie hine on poet swearte fenn læddon 7 hine on pa horwihtan wæter wurpon 7 besencton. 
Goodwin s. 34 :

pa on pære nibte stilnysse zelamp semninza, pæt pær comon mycele meniu para awyrizedra zasta, and hi eall pæt hus mid heora cyme fyldon; and hi on ealce healfe inzuton ufan and neoðan and eghwonen. Hi wæron on ansyne ezslice and hiz Læfdon mycele heafda, and langne sweoran, and manizre ansyne: hi wæron fulice and orfyrme on heora beardum; and hi hæfdon and ruze earan and hi hæfdon woh nebb

and re內elice eazan, and fule muðas; and heora topas wæron zelice horses twuxan; and him wæron pa protan mid leze zefylde, and hi wæron on gristlice on stefne: hi hæfdon woze sceancan and mycele cneowu and hindan zreate, and miscrocetton tan and has runizendum stefnum; and hi pa swa ungemetlicre zestundum foron and swa unmetlicre eze, pæt him puhte pæt hit eall be tweox heofone and eorðan hleoprode pam ezeslicum stefnum. Næs pa næniz yldend to pam pæt syppan hi on pæt hus comon hi pa sona pone halzan wer eallum limum zebundon, and hi hine tuzon and læddon ut of pære cytan, and hine pa læddon on pone sweartan fenn and hine pa on pa orwehtan wæter bewurpon and besencton.

Dịe geschichte wird dann weiter erzählt, wie in der Cottonhandschrift, bis zum erscheinen des Bartholomæus und entspricht der darstellung dort. Nach der stelle, welche sich bei Goodwin s. 40, z. 10 findet, ist plötzlich ein schluss im Vercellibuche darangesetzt.

$135 \mathrm{~b}$ :

7 pa æfter pam fleah se haliza zuòlac mid

pain apostule sce bartholomei (bartholoma?) to heofona rices wuldre 7 hine se hælend pær onfenz 7 he pær leofad 7 rixap In heo fona rices wuldre a butan ende on ecnesse amen finit: 7

Goodwin benutzte das Vercellibruchstuck, wenn auch in ungenitgender weise. B. Thorpe überliess ihm eine abschrift (wol die von Blume gefertigte?). Goodwin sagt von dem Vercellibruchstücke:

'It is curious, as presenting a text very different from the Cottonian copy; indeed it has almost the appearance of being part of an independent translation, though I believe this is not really the case'.

Eine vergleichung scheint mir sicher zu stellen, dass das Vercellibuch keine andre bearbeitung der legende enthält, sondern, abgesehen von kleinigkeiten, denselben text wie die Cottonhandschrift bietet. Der schluss wurde vom schreiber willkürlich darangesetzt, an einer stelle, die ihm ungefähr passend erschien.

Ich lasse zum schlusse die 13. homilie, wovon sich ein teil bei Cooper facsimiliert findet, nun vollständig folgen. 
$75 \mathrm{~b}$ : Spel to priddan zanzdæze. ${ }^{\prime}$

ĐIS IS ${ }^{2}$ se pridda dæz men pa leofestan pysse tide pe us on swiðe wel zelimpeð pæt we ealle eaðmodlice sculon dryhtne peowian 7 wel forð zelæstan poet we nu ær on pyssum dazum lærde wæron bið pat swiðe herizendlic pæt man on pone zodan siðfæt zodes be boda freolice fere 7 zanze 7 ne læte hine aslacian halizra dæda for pon pe dryhten jus cwæp 7 zehet pam fæstrædan men 7 p $\bar{a}$ purh wunizendan In zodes willan; quod ${ }^{3}$ aut perseuerauerit usque In fin $\bar{e}^{4}$ saluus erit. se man sede ${ }^{4}$ purhwunad In zodum dædum oo lifes ende he bio hal zeworden . swa pænne swide ${ }^{4}$ wel zelimplic is pæt we pysne dæz zedeflice bezanzen mid heofenlicū mæzenum ${ }^{4}$ syndon pas pry dazas to eacan odrum pinzum forpan us zesette pe we lifiende sien 7 zif we mid ures lichaman lustum hwæt zimeleaslices dydon on pyssum feowertezum nihtum wið zodes willan bete he pæt on pyssum dazum nu 7 clænsize hine pæt he mæze beon pys merzen lican dæze æt pære halzan dryhtnes upafaznes tide clæne æt dryhtnes wiofode $7 \mathrm{pær}$ onfon weddes pæs ecan rices . poet is cris tes sylfes lichoma 7 his blod ${ }^{4}$ pæt we nu nemnap husl seman sede

$76^{\mathrm{a}}$ :

unnyttan ${ }^{5}$ lustas druncennesse 7 fyrenlusta zytsunz 7 unriht $^{6}$ zestrodu hwæt wunap pysses mid Jam men ofer hydum In Jære byrzenne nempe das seonuwa 7 para bana dust In pære eorðan zewiteðे swa swa zlidende scuwa pær pæt la zewioròan meahte pæt $\partial a$ drizan ban sprecan meahton of خære byrzenne to $\partial a m$ men pe hie swa ziorne behealde $\partial$ hie cwædon ponne uus tohwan la ju earma man 7 pu unzesæliza zymest ou pysse worulde swa swið odje tohwan bezæst

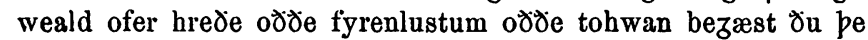
Jam wælhreowestan hlaforðum pæt is hleahtru 7 uncystum be heald me 7 sceawa mine ban 7 ondræd pe pinne fyrenlust 7 pine zytsunze pæt $\partial u$ eart $n u$ pæt ic wæs io pæt ic eom nu pæt $\partial_{u}$ wiorठ̀est eft peah pe In me purhwunode idelnes 7 fyrenlust ne for nimðe næfre unrihtwisnes peah je me fyrenlust zewemde zefræte wape mid clænnesse zeseohðu me In duste formolsnod, $\mathrm{e}^{\mathrm{7}} 7$ burh pæt forlæt du pinne pone yfelan lust dus cleopedon pa ban to us zif hie sprecan meahton of pære byrzenne 7 we mazon huru onzytan pa bysene peah pe hie swizien on pam for ${ }^{\circ}$ ewite num ure azenre tweonunze emne us zewioröed eft se ilca dead pæt he us adrife $\delta$ of eardunza usses lichoman Utan we nu

1 In roter schrift.

2 In roter schrift. Das erste IS steht im $\boxplus$.

3 So hat die hs.

4 Im facsimile bei Cooper sind öfters buchstaben ausgestrichen und über die zeile geschrieben, in der hs. stehen dieselben ohne korrektur in der zeile.

5 Am anfange der zeile sind zwei löcher im pergamente.

6 Zwischen un und riht loch.

7 So die hs. 
py ziornlicor hine zepencan pone deað pe we hine ær witon ær pan pe he færinza cume. forpan he cumed on us unzepinzod forpan zod wolde pæt pam men wære his ytemesta dæz uncuð pæt he hine forpan to pan zezearwode butan ænizre ablin

$76^{\mathrm{b}}$ :

nednesse pæt biò se ezeslica dæz 7 se ondrýsenlica on " Jam dæze dryhten scrifeð manna zehwylcum ece lif swa in heofena rices hyhðo swa on helle witū swa he nu her on worulde zecarnizan wile tilizen we nu forpan panne us zod forzife pæt we his pa halzan lare zehyren swa we nu dydon pæt we syn pe beteran 7 pe selran for bæm lifum in ealra worulda world to widan feore mid fæder 7 mid suna 7 mid pam balzan zaste In ecnesse Amen.

Ein vergleich dieser 'homilien mit ähnlichen ergibt, dass sich verschiedene auch anderweitig finden. So stehen no. 4 und no. 18 bei Wanley, Catal. s. 114 und s. 45 angeführt No. 3 kann der s. 13 erwähnten entsprechen. Doch da ich darauf an andrem orte ausfuhrlicher zurtickkomme, gentige hier die andeutung.

LEIPZIG.

R. P. Wuelcker.

${ }^{2}$ Hier auf der riickseite zeigen sich widerum die zwei löcher im pergamente. 